\title{
The Effectiveness of Weekly Supervised Homework Sessions in an Aerospace Structural Mechanics Course
}

\section{Mr. Waterloo Tsutsui, Purdue University - West Lafayette}

Waterloo Tsutsui is a Ph.D. candidate in the School of Aeronautics and Astronautics at Purdue University in West Lafayette, Indiana. Prior to attending Purdue University, he was an engineer in the automotive industry for 10 years. In the most recent industrial position, he was a senior mechanical engineer at Johnson Controls, Inc. in Milwaukee, Wisconsin. He is a licensed Professional Engineer in the states of Michigan, Wisconsin, and Indiana.

\section{Dr. Michael C. Loui, Purdue University - West Lafayette}

Michael C. Loui is the Dale and Suzi Gallagher Professor of Engineering Education at Purdue University. He was previously Professor of Electrical and Computer Engineering and University Distinguished Teacher-Scholar at the University of Illinois at Urbana-Champaign. His interests include computational complexity theory, professional ethics, and engineering education research. He serves as Editor of Journal of Engineering Education and as a member of the editorial boards of College Teaching and Accountability in Research. He is a Carnegie Scholar and an IEEE Fellow. Professor Loui was Associate Dean of the Graduate College at Illinois from 1996 to 2000. He directed the theory of computing program at the National Science Foundation from 1990 to 1991. He earned the Ph.D. at M.I.T. in 1980. 


\title{
The Effectiveness of Weekly Supervised Homework Sessions in an Aerospace Structural Mechanics Course
}

\begin{abstract}
AAE 35200 is an aerospace structural mechanics course for third-year students majoring in Aeronautical and Astronautical Engineering at Purdue University. In AAE 35200, students were given the opportunity to attend weekly optional one-hour supervised homework sessions instructed by a graduate teaching assistant (TA) during the Fall 2015 semester. The contents of the supervised homework session included the theoretical background and technical details about the assigned homework problems, knowledge beyond the original context of the problems, and alternative approaches to solve the problems. In this study, we investigated the effect of the supervised homework sessions on students' homework and exam scores. We found that the supervised homework sessions had the most influence on the homework scores of the students with low native ability. Also, as the byproduct of the study, we investigated the effect of the Scholastic Aptitude Test (SAT)-Math scores on students' academic performance. We found that the SAT-Math score was not correlated with the exam score. In order for us to rigorously study the effect of student attributes (independent variables) on the academic performance (dependent variables), we performed an analysis of covariance (ANCOVA) on the experimental data, controlling for student attributes. We found that the attendance and interaction between SATMath and attendance were the two terms that influenced the homework scores the most.
\end{abstract}

\section{Introduction}

In courses in science and engineering, ancillary learning opportunities outside the classroom can take different forms. Currently, two popular ancillary approaches are peer-led team learning (PLTL) and supplemental instruction (SI). In a PLTL session, students solve problems as a group that is led by a peer leader who is not normally a subject matter expert (SME), whereas an SI session is led by an SME. ${ }^{1}$ In both types of sessions, students learn how to solve problems that are similar to the materials that are covered in the lecture. By contrast, organized sessions on the assigned homework problems by teaching staff have not been seen widely. For that reason, understanding the effectiveness of this particular form of outside learning is lacking.

There are advantages to both students and teaching staff on the use of homework problems during these ancillary sessions. From the viewpoint of students, they must use time efficiently since they are constantly under pressure to complete homework assignments for multiple classes during a semester. For this reason, students can motivate themselves to take advantage of one hour per week of the supervised homework session simply because the benefits to the students are tremendous. For instance, the students can learn how to set up a problem and how to approach the problem differently. Further, the assigned homework counts toward the students' course grade. From the viewpoint of teaching staff, there is no need to prepare extra lecture material because they have already prepared a solution to the assigned problems, which can be utilized to provide necessary information during the session. Limiting the session to one hour per week is convenient for the teaching staff members since they are also under pressure to conduct their research outside their teaching duties. 
In this scholarship of teaching and learning (SoTL) study on the effectiveness of the supervised homework sessions, we first reviewed the relevant work in peer-led team learning (PLTL) and supplemental instruction (SI). Then we compared the homework and exam scores against the students' attendance to the homework sessions. After that, we isolated the effect of attendance from the effects of the other independent variables using a quantitative statistical assessment method, ANCOVA. As the final part of the study, we briefly analyzed qualitative data from a student survey. We sought to answer the following research question: Did the students who participated in the supervised homework sessions perform better than those who did not participate?

\section{Literature Review}

\section{Assessment of Optional Study Sessions outside the Classroom}

In this section, we provided the review of PLTL and SI, so that we can differentiate these approaches from our TA-led supervised homework session approach later in this article.

PLTL is a popular structure for learning opportunities outside the classroom. In PLTL, a small group of 6 to 8 students work as a team to solve problems. The group of students is led by a peer-leader who facilitates the discussion. Since the objective of the group leader is to facilitate conversation, not to give technical feedback, a PLTL session can take place without a subject matter expert (SME). Therefore, PLTL can utilize many undergraduates as peer leaders. As a result, a large number of students can experience small group activities in a session. Loui et al. ${ }^{1}$ reported that in an undergraduate electrical engineering course, regular attendance at PLTL sessions improved the students' understanding of the course materials, thus resulting in the significantly higher scores in final exams. Similarly, for an undergraduate organic chemistry course, regular attendance in PLTL sessions produced significantly improved student academic performance and resulted in higher motivation toward the course work. ${ }^{2}$ For an undergraduate biology course, Born et al. ${ }^{3}$ analyzed the effectiveness of PLTL for underrepresented minority and women in science. The research concluded that PLTL had positive impact on their academic performance. For a first-semester undergraduate general chemistry course, Hockings et al. ${ }^{4}$ concluded that students who regularly participated in PLTL sessions received higher grades than those who did not participate. The difference was about one-third of a grade point, for instance, B vs. B-.

PLTL became well-established because PLTL could be modified to suit the needs of the students and educators. For instance, PLTL might also be conducted during a regular lecture session. Brown and Poor ${ }^{5}$ investigated the effectiveness of in-class peer tutoring (ICPT). In ICPT, a peer team leader acts as a teaching assistant for a small group of students during the in-class exercise. The authors reported that more than $80 \%$ of students liked the ICPT experience. Furthermore, PLTL allowed students to experience social benefits. Students who participated in PLTL regularly made new friends during the sessions, and these peers became a helpful source of knowledge. ${ }^{1}$ In addition, PLTL could positively affect the first-year students or certain underrepresented students. PLTL is typically implemented in courses for first-year undergraduate students. Therefore, the benefits of PLTL reported in previous research might not 
accrue to more mature students, such as third- and fourth-year undergraduate students and graduate students.

SI is another popular form of organized learning outside the classroom. ${ }^{6,7,8}$ In SI, unlike PLTL, a group of students is led by an SME, usually an advanced graduate student or an instructor. Due to the limited supply of qualified SMEs, the number of students in an SI session tends to be relatively larger.

The learning objectives of PLTL and SI are the same: to enhance the higher-level understanding of the subject matter through the additional study sessions outside the classroom. PLTL and SI take different paths to reach this objective. Because of this difference, SI may be conducted as a lecture-based approach, as opposed to the team-based approach of the PLTL. In fact, Prince ${ }^{9}$ indicated that the effective coursework does not necessarily mean a team-based study.

Both SI and our homework sessions were led by an SME. However, outside-the-classroom learning opportunity is different because the assigned homework from lecture is used as the materials, whereas SI leaders prepare their own materials. This difference distinguishes the supervised homework session from SI.

\section{Research on Teaching Mechanics of Materials}

The subject matter of our research was structural mechanics in aircraft. Few articles have been published that report scholarship of teaching and learning (SoTL) research specifically to the mechanics of materials. Montfort et al. ${ }^{10}$ investigated students' conceptual understanding of bending stress and shear stress. The research concluded that there was not much difference between the undergraduate and graduate students in the conceptual understanding of these structural mechanics topics although graduate students exhibited better computational skills than undergraduate students. Similar to the study by Montfort et al., ${ }^{10}$ Brown et al. investigated how students understood the states of stress in general cases in mechanics of materials ${ }^{11}$ as well as the cases in beams. ${ }^{12}$

The studies of Montfort et al. ${ }^{10}$ and Brown et al. ${ }^{11,12}$ focused on the certain topics of mechanics of materials (e.g., normal and shear stresses in beams). The previous work did not address the teaching topics specific to AAE 35200 at Purdue University, such as shear flow and shear center, which are specific to the thin-walled structures of an aircraft fuselage and wings. ${ }^{13}$ In AAE 35200 at Purdue University, students had already completed the beam analysis in the prerequisite course before taking AAE 35200.

\section{Controlling for Student Aptitude using ACT Scores}

Thomson and Zamboanga ${ }^{14}$ discussed how students' academic aptitude (measured by American College Testing, ACT, scores) affected the academic performance in the study of psychology. Although the subject area in psychology was different from our SoTL research in engineering, the similar research procedures could be applied to the proposed research by controlling for the general aptitude in order to understand the pure effectiveness of our supervised homework sessions. 
In our research, one of the most significant covariates was the SAT-Math scores. To demonstrate this concept, let us consider the following hypothetical scenario: We found the students who participated in the supervised homework sessions performed better in the exams than those who did not participate in the session. However, the differences in the exam grades might have been based on the fact that those who participated in the supervised homework sessions had higher native aptitude to solve mathematical problems. In this case, since the starting point was different in each participating student, it was impossible to make conclusion on the effectiveness of the supervised homework sessions. As a consequence, in this study, we controlled for aptitude by using SAT-Math scores. It was also possible to use other scores, such as grade point average (GPA) from high school and previous university courses, to control for the students' native aptitude. We, however, did not pursue these options since the high school and previous university courses might have contain a large number of courses that were not related to engineering and mathematics.

\section{Course Context}

\section{Descriptions of the Course}

AAE 35200 is a course in aerospace structural mechanics at Purdue University. During the Fall 2015 semester, 109 undergraduate students took the course. The course had two lecture sections: 62 students took the Monday-Wednesday-Friday section and 47 students took the TuesdayThursday section. The durations of a lecture in each section were 50 minutes and 75 minutes, respectively. The same instructor delivered the lectures in both sections, presenting the same content. In addition, the graduate teaching assistant (TA) conducted an optional one-hour supervised homework session every Wednesday evening. The TA is the researcher for this study who is a fifth year Ph.D. student majoring in structures and materials in the School of Aeronautics and Astronautics at Purdue University.

As previously noted, the difference between our activities and PLTL/SI was that our TA-led supervised homework session used the assigned homework problems from the lecture to learn concepts on structural mechanics, whereas the previously-reported projects used supplemental materials to teach the materials. More specifically, in our research, a problem was discussed by the TA using a document camera and projector. Since no small group activities were involved, the TA-led supervised session was not a cooperative learning session.

As the TA led the session by showing the key concepts needed to solve the problems, the TA continually asked questions on the important ideas to the participating students. In return, the students replied and also asked questions whenever they felt necessary. When the TA felt a question from the students was worth discussing, the question was returned to the entire students and discussed together to seek the answers. All key procedures and key answers were discussed. As a result, when the students finished the session, they had the clear directions on how to solve the homework problems.

One homework problem required the analysis of stress state to calculate principal stresses and their directions. In this case, the TA demonstrated the use of the matrix method (i.e., eigenvalues and eigenvectors) to solve the problem. Then, the TA demonstrated the use of the Mohr's circle 
method to confirm that the calculated principal stresses and directions were the same in the two different methods.

\section{Descriptions of the Participants}

Out of 109 students, 58 students completed consent forms to participate in the SoTL study. Table 1 shows the demographics of the participants.

Table 1: Demographics of Participants

\begin{tabular}{|c|c|c|c|c|}
\hline Gender & Student Status & $\begin{array}{c}\text { English Language } \\
\text { Speaker }\end{array}$ & $\begin{array}{c}\text { Number of } \\
\text { Students }\end{array}$ & $\begin{array}{c}\text { Percentage of } \\
\text { Students }\end{array}$ \\
\hline Female & $\begin{array}{c}\text { Out-of-State } \\
\text { Domestic }\end{array}$ & Native & 4 & $7 \%$ \\
\hline Female & International & Non-Native & 2 & $3 \%$ \\
\hline Male & $\begin{array}{c}\text { In-State } \\
\text { Domestic }\end{array}$ & Native & 20 & $35 \%$ \\
\hline Male & $\begin{array}{c}\text { Out-of-State } \\
\text { Domestic }\end{array}$ & Native & 24 & $41 \%$ \\
\hline International & Native & Non-Native & 6 & $10 \%$ \\
\hline
\end{tabular}

\section{Methods}

The purpose of the SoTL study was to understand the effect of the supervised homework sessions on the students' academic performance. To begin the SoTL project, the Institutional Review Board (IRB) approval was obtained at Purdue University. Also, the TA took attendance at every supervised homework session by circulating a pre-printed spreadsheet to the students. The attending students generally stayed from the beginning to the end of each session. If, however, some students chose to leave after spending some time in the room, they were considered to have attended the session.

A survey for the SoTL project was administered during the eighth week of the semester. Since the lecture for the Fall 2015 semester lasted for 16 weeks, the timing for the survey was exactly at the half-way point of the semester, which conformed to the timing suggested by Lewis. ${ }^{15}$ The following were the survey questions: Name, gender, nationality (domestic or international), instate or out-of-state for domestic students, whether or not English is the first language, the scores of SAT-Math (200 - 800) and ACT-Math (1-36), and qualitative questions about the reasons for attending or not attending the supervised homework sessions regularly (more than or equal to $50 \%$ attendance). One of the main reasons for the use of the survey was to see if students with certain demographic backgrounds (e.g., gender, domestic/international student status, and English language nativity) benefited from the supervised homework sessions more than others. 
The SAT-Math scores were used to control for the native aptitude of the students to solve mathematical problems. When a student provided both SAT-Math and ACT-Math scores, the SAT-Math score were used; the ACT-Math score was disregarded in this case. When a student provided only an ACT-Math score, the ACT-Math score was converted to the equivalent SATMath score using established guidelines. ${ }^{16}$ We hypothesized that those students who attended the supervised homework sessions performed better than those who did not attend. As we wanted to evaluate the research hypothesis rigorously, we isolated the effect of the session participation from the effects of the other variables using ANCOVA.

We used the SAT-Math score as the indicator of students' native aptitude for solving engineering problems. More specifically, students' academic performance was adjusted by controlling for their SAT-Math scores in addition to a multivariate linear regression using ANCOVA.

ANCOVA allowed us to mitigate the effect of covariates from the experimental results. In other words, when analyzing the final academic performance, we placed all students at the same starting line by controlling for the SAT-Math scores in order to see the pure effect of the session participation and to evaluate our research question, rigorously. To analyze the experimental data, we classified the students into three groups: High, medium, and low SAT-Math groups (Table 2). The average and standard deviation of SAT-Math score of the 58 participating students were 739.7 and 50.5, respectively.

Table 2: Classification of Participated Student based on SAT-Math Score

\begin{tabular}{|c|c|c|c|}
\hline & Low SAT-Math & Medium SAT-Math & High SAT-Math \\
\hline SAT-Math Scores & $600-710$ & $720-770$ & $780-800$ \\
\hline Number of Students & 18 & 19 & 21 \\
\hline
\end{tabular}

After we conducted the survey in the eighth week of the semester, the survey sheets were placed inside sealed envelopes. Since the surveys included the names, the researchers did not open the envelopes until after the submission of course grades in December 2015. This procedure prevented coercion influence on course grade. In fact, all students were assured that the data would not be used until after the course grades were filed.

\section{Results}

In this section, the participating students' performance is evaluated based on their average homework scores from Homework 1 - 13 (Fig. 1) and average exam scores from Exams 1 - 3

(Fig. 2). We did not take the final grades for the class into consideration since the final grades are determined by the composite of exam and homework scores (i.e., 25\% Exam 1, 25\% Exam 2, 25\% Exam 3, and 25\% HW), and the final grades will simply be the weighted average (i.e., 75\% Exams and 25\% homework) of our research result. Tables 3 and 4 show the range of student performances, where the sample population is, again, divided into high, medium, and low SATMath groups. Furthermore, the sample population is divided into two groups based on the attendance to the supervised homework sessions, where we use the $50 \%$ participation to the supervised homework session as the transition point. 


\section{Effect of Attendance at Supervised Homework Sessions}

To determine whether students who attended the supervised homework sessions performed well in the homework score, we conducted a linear regression analysis, separately for each SAT-Math group. Fig. 1 shows the relationship between the homework score and homework session attendance for each group. The upward trend with positive slopes is clear to see in Fig. 1. The strong upward trend is especially true for the students whose SAT-Math score is low, while the effect of the session attendance becomes less as the SAT-Math score becomes higher. The slopes in the Fig. 1 can confirm this observation as the slopes of $0.11,0.12$, and 0.27 are observed for the students with high SAT, medium SAT, and low SAT-Math scores.

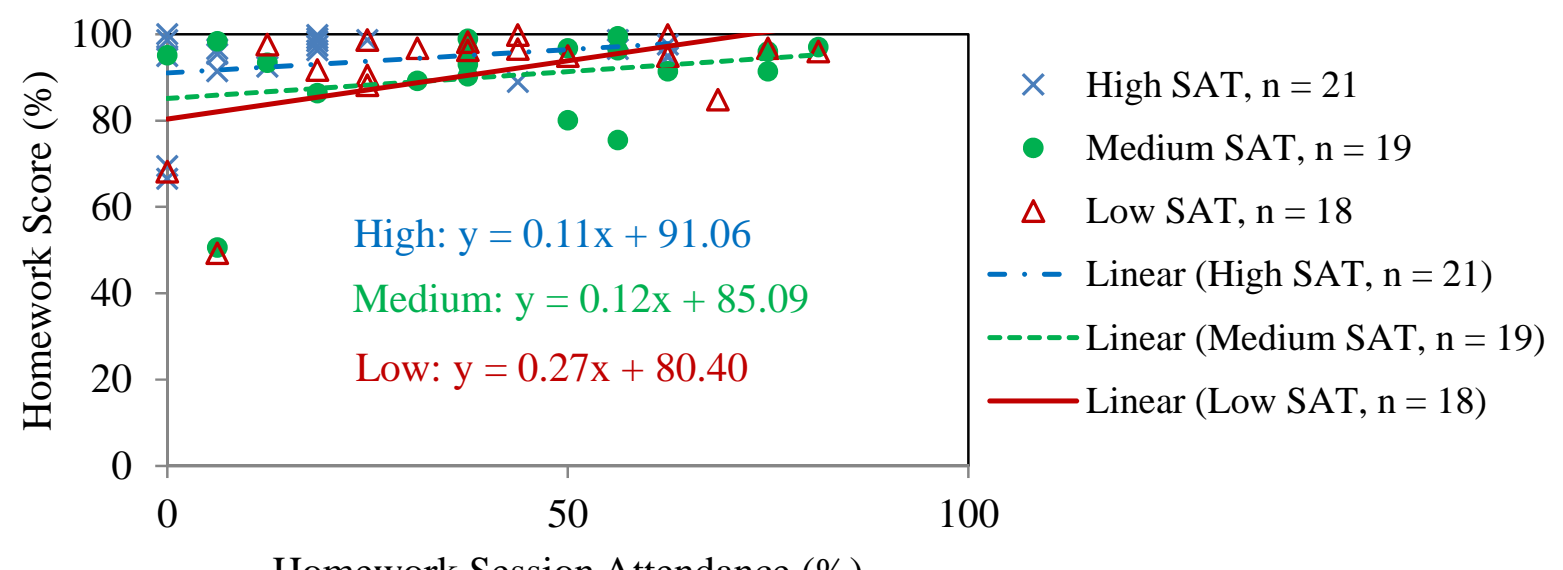

Homework Session Attendance (\%)

Figure 1: Homework Score vs. Homework Session Attendance

Table 3 shows the range of students' homework results. Again, the sample population is divided into the high, medium, and low SAT-Math groups as well as divided into two groups based on the homework session attendance. Table 3 indicates that a) the maximum scores are about the same in all three SAT classifications, b) the minimum scores follows the trend of SAT scores, where high SAT results in high minimum score, c) 50\% or more attendance results in higher homework score in all three SAT score classification, and d) differences between $50 \%$ or more attendance and the less than 50\% attendance is the largest at the low SAT group (5.3\%).

Table 3: Distribution of Homework Score

\begin{tabular}{|c|c|c|c|c|}
\hline & $\begin{array}{c}\text { Number of } \\
\text { Students }\end{array}$ & $\begin{array}{c}\text { Max / Min } \\
\text { Scores }\end{array}$ & $\begin{array}{c}\text { Average Score } \\
\text { (Attendance } \geq 50 \%)\end{array}$ & $\begin{array}{c}\text { Average Score } \\
\text { (Attendance < 50\%) }\end{array}$ \\
\hline High SAT & 21 & $100 / 66.5$ & 96.4 & 92.7 \\
\hline Medium SAT & 19 & $99.5 / 50.5$ & 91.5 & 89.1 \\
\hline Low SAT & 18 & $99.7 / 49.2$ & 94.5 & 89.2 \\
\hline
\end{tabular}

Fig. 2 shows the relationship between the exam score and homework session attendance for three SAT groups. The linear regression lines for all three SAT groups show a slope of almost zero, which indicates that attendance at the supervised homework session participation does not affect 
exam scores. In fact, the data of the exam score seems to be scattered uniformly, paralleling to the horizontal axis.

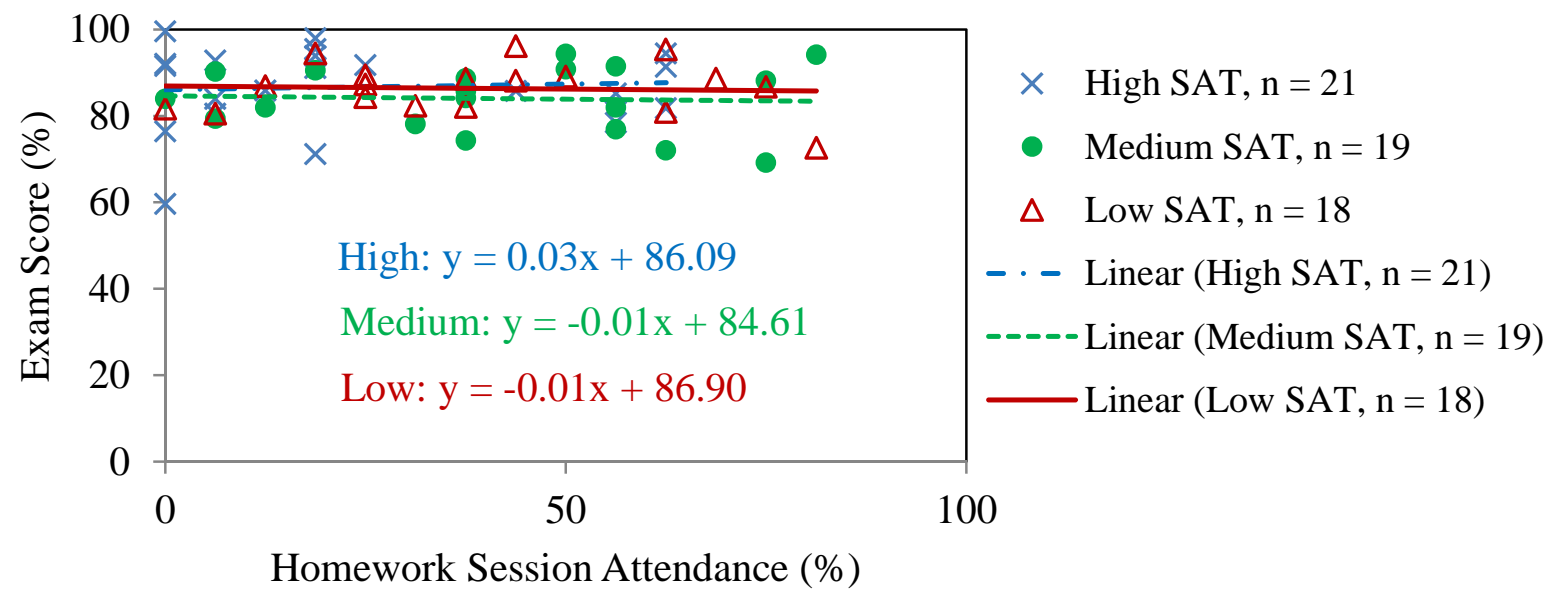

Figure 2: Exam Score vs. Homework Session Attendance

Based on the comparison between average scores in Tables 3 and 4 for each SAT group, the exam scores show fewer differences than the homework scores. The average scores for the two groups based on the session attendance are identical in Table 4. Therefore, the conclusion is that the supervised homework session has less impact on students' exam scores than the homework scores.

In Fig. 3, the slopes for the medium and low SAT groups are negative although they are not significantly different from zero. This is also partially confirmed by Table 4, where the low SAT group shows that the students in this group perform worse in exam with higher attendance when they are compared against those with lower attendance. However, since the lines are almost horizontal in Fig. 2, it is safe to assume that the effect of the homework session attendance is negligible on the exam score.

Table 4: Distribution of Exam Score

\begin{tabular}{|c|c|c|c|c|}
\hline & $\begin{array}{c}\text { Number of } \\
\text { Students }\end{array}$ & $\begin{array}{c}\text { Max / Min } \\
\text { Scores }\end{array}$ & $\begin{array}{c}\text { Average Score } \\
\text { (Attendance } \geq 50 \%)\end{array}$ & $\begin{array}{c}\text { Average Score } \\
\text { (Attendance < 50\%) }\end{array}$ \\
\hline High SAT & 21 & $99.6 / 59.6$ & 86.2 & 86.8 \\
\hline Medium SAT & 19 & $94.3 / 69.1$ & 84.3 & 83.7 \\
\hline Low SAT & 18 & $96.2 / 72.6$ & 85.5 & 86.8 \\
\hline
\end{tabular}




\section{Effect of SAT Scores}

Figs. 3 and 4 show the homework and exam scores based on the SAT-Math score. The data in both Figs. 3 and 4 results in the positive slope meaning that the higher SAT-Math score results in the higher academic performance. However, when the slopes of the regression models are compared between Fig. 3 and 4, the slope in Fig. 4 is much smaller. In fact, the slope is almost zero, which indicates that the SAT-Math score is not correlated with exam scores.

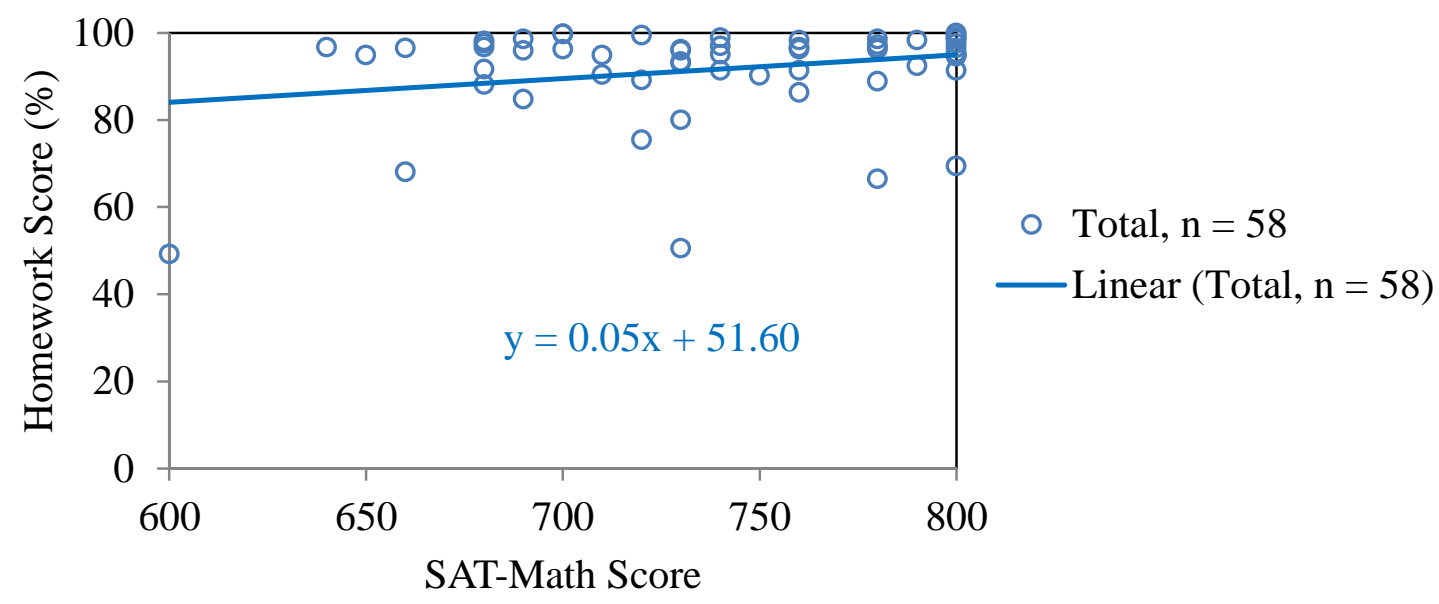

Figure 3: Homework Score vs. SAT-Math Score

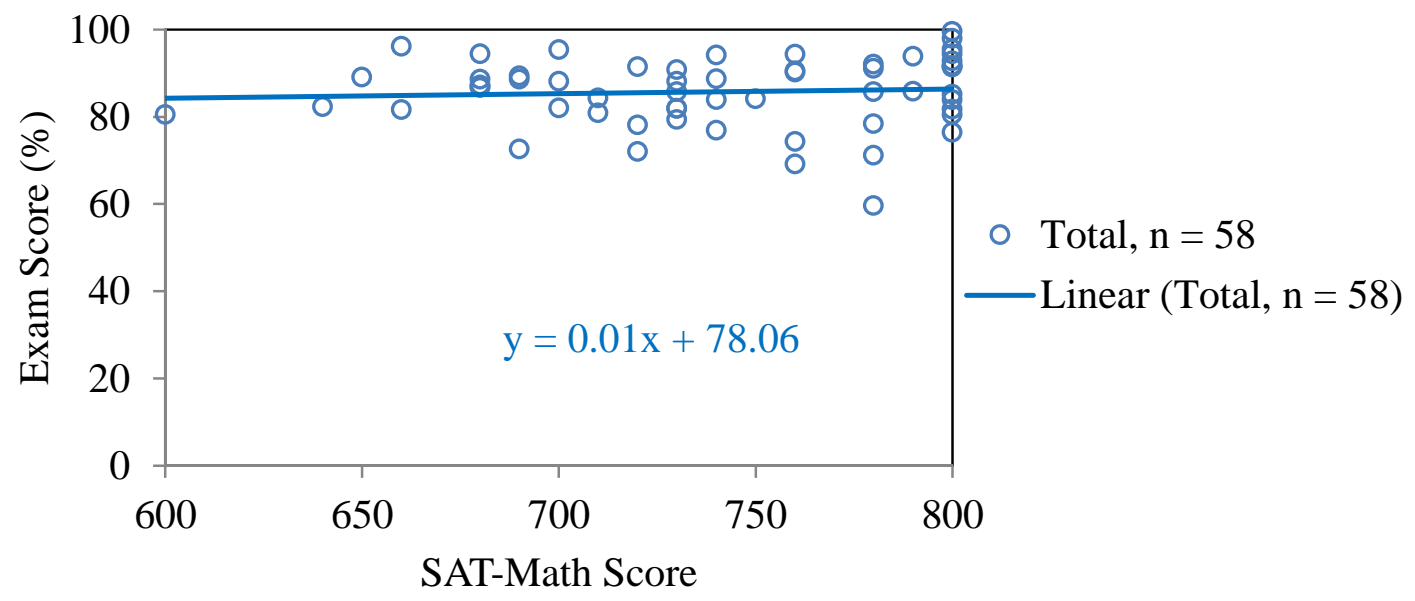

Figure 4: Exam Score vs. SAT-Math Score

\section{ANCOVA}

We conducted an ANCOVA on the experimental data using Minitab 17. As a prerequisite, we conducted normality study on the exam and homework scores. We found the P-values of 0.147 and less than 0.005 for exam and HW scores, respectively. These P-values indicate that the exam scores are normally distributed; however, the homework scores are not normally distributed due to the existence of outliers. The non-normal data means that the ANCOVA may 
not be the most informative statistical tool for the analysis of the homework scores. Despite this, we chose to pursue ANCOVA as we are currently gathering more data in the current semester, and we are hoping that the larger sample size will reduce the effect of the outliers that exist in our data set.

Equations 1 and 2 indicate the regression models based on our experimental data, controlling for student attributes, such as normalized SAT-Math score using z-scores, where z-score = (observed data - average) / standard deviation. The regression models indicate not only the influence of covariates (e.g., 2.74 SAT) but also the influence of the interactions (e.g., 2.94 SAT*Attendance). Table 5 shows the discrete variables, observed number of students $n$, constants $\beta_{0}$, and $\gamma_{0}$ in Equations 1 and 2. Immediately after Table 5, the detailed information on three terms (i.e., responses, factors, and covariates) is provided since the definition of these terms is essential in our ANCOVA study.

Homework Score $=\beta_{0}+2.74$ SAT +3.35 Attendance - 2.94 SAT*Attendance (Equation 1) Exam Score $=\gamma_{0}+0.56$ SAT -0.18 Attendance -0.37 SAT $*$ Attendance

Table 5: Discrete Variables, Number of Students, and Constants for Regression Equations

\begin{tabular}{|c|c|c|c|c|c|}
\hline \multicolumn{2}{|c|}{ Discrete (Categorical) Variables } & \multirow{2}{*}{$n$} & \multirow{2}{*}{$\beta_{0}$} & $\gamma_{0}$ \\
\cline { 1 - 2 } Gender & Status & Language & & 94.02 & 89.55 \\
\hline 0 & 0 & 0 & 0 & 90.60 & 99.65 \\
\hline 0 & 0 & 1 & 0 & 96.83 & 92.84 \\
\hline 0 & 1 & 0 & 4 & 93.40 & 102.94 \\
\hline 0 & 1 & 1 & 0 & 98.98 & 80.87 \\
\hline 0 & 2 & 0 & 0 & 95.60 & 90.97 \\
\hline 0 & 2 & 1 & 2 & 89.44 & 84.03 \\
\hline 1 & 0 & 0 & 20 & 81.51 & 90.43 \\
\hline 1 & 0 & 1 & 0 & 92.25 & 87.31 \\
\hline 1 & 1 & 0 & 24 & 84.31 & 93.72 \\
\hline 1 & 1 & 1 & 0 & 94.40 & 75.34 \\
\hline 1 & 2 & 0 & 2 & 86.46 & 81.75 \\
\hline 1 & 2 & 1 & 6 & & \\
\hline
\end{tabular}


Responses: Dependent Variables

Homework Score:

Expected homework score in percent (\%)

Exam Score:

Expected exam score in percent (\%)

Factors: Discrete (Categorical) Independent Variables

Gender:

$0=$ Female $(\mathrm{n}=6)$

$1=$ Male $(\mathrm{n}=52)$

Status:

$0=$ In-state domestic student $(\mathrm{n}=21)$

1 = Out-of-state domestic student $(\mathrm{n}=27)$

2 = International student $(\mathrm{n}=10)$

\section{Language:}

$0=$ English as a first language (50)

1 = English as a non-first language (8)

Covariates: Continuous Independent Variables

\section{SAT:}

Z-values corresponding to the SAT-Math score of each participating students were calculated based on the average and standard deviation of 739.8 and 50.5, respectively. The maximum and minimum z-values were 1.192 and -2.769 corresponding to the observed maximum and minimum SAT-Math scores of 800 and 600, respectively.

\section{Attendance:}

Z-values corresponding to the supervised homework session attendance (\%) were calculated based on the average and standard deviation of $34.3 \%$ and $24.7 \%$, respectively. The maximum and minimum z-values were 1.906 and -1.390 corresponding to the observed maximum and minimum attendance of $81 \%$ and $0 \%$, respectively.

In order to further clarify variables and interactions that have significant impact on the response, the p-values for the variables were recorded in Table 6. With the assumption of the 95\% confidence interval, the p-value of less than 0.05 would indicate the statistical significance, thus leading to the rejection of null hypothesis. In our ANCOVA analysis, the terms that had the pvalue less than 0.05 were the attendance $(p=0.021)$ for the homework score and interaction term SAT*Attendance $(p=0.044)$ for the homework score as shown in Table 6: the coefficients of both Attendance and SAT*Attendance in Equation 1 are significantly different from zero. This observation confirms our earlier observation. 
Table 6: P-Values for Variables

\begin{tabular}{|c|c|c|}
\hline Variables & P-Value (Homework) & P-Value (Exam) \\
\hline SAT & 0.082 & 0.634 \\
\hline Attendance & $\mathbf{0 . 0 2 1}$ & 0.864 \\
\hline Gender & 0.169 & 0.055 \\
\hline Status & 0.611 & 0.071 \\
\hline Language & 0.519 & 0.220 \\
\hline SAT*Attendance & $\mathbf{0 . 0 4 4}$ & 0.734 \\
\hline Gender*Language & 0.645 & 0.625 \\
\hline
\end{tabular}

Note: Minitab removed the interactions Gender*Status and Status*Language from the analysis since these terms could not be estimated.

We also examined multiple R-squared in order to evaluate how well the generated regression models fit the data. We obtained the R-squared values of $29.64 \%$ and $20.13 \%$ for homework and exam scores, respectively (Table 7). The fact that the multiple R-squared is higher in the homework score makes sense because the two p-values for the variables for homework was found to be statistically significant as shown in Table 6 under the assumption of 95\% confidence interval, whereas no such case was observed for the exam score. Based on the obtained Rsquired values, we conclude that the generated regression models in our study can explain 20 $30 \%$ of the variation in the homework and exam scores.

Table 7: Multiple R-squared

\begin{tabular}{|c|c|c|}
\hline & Homework Score & Exam Score \\
\hline Multiple R-squared & $29.64 \%$ & $20.13 \%$ \\
\hline
\end{tabular}

\section{Discussion and Implications}

The results in Fig. 1 show that attendance at the supervised homework sessions improved students' homework scores. However, as Fig. 2 indicate that the sessions did not result in the noticeable difference in the exam score. That is, the session attendance had less impact on students' exam scores than on their homework scores. Furthermore, the SAT-Math scores, combined with the session attendance, appeared to have minor influence on the homework scores (Fig. 3) but not on the exam scores (Fig. 4).

Based on the experimental results, we can argue that students with low SAT-Math scores appeared to benefit from attending the supervised homework session. Therefore, we were able to answer the research question provided at the end of the introduction section "Did the students who participated in the supervised homework sessions perform better than those who did not participate?" as follows: Yes, the students who attended the homework session performed better on the homework than students who did not; however, the effect was limited only to those 
who did not perform well in SAT-Math. On the other hand, those students with high SAT-Math scores were able to perform well with or without attending the supervised homework sessions.

Another interesting finding was that the SAT-Math score was not correlated with exam scores. In other word, SAT-Math scores were not the accurate measure to predict the exam scores in the research that we conducted. This conclusion seemed to be contrary to the established opinion on the SAT-Math as an excellent predictor of academic success in college. ${ }^{17,18}$ To this end, we presume that perhaps the students with lower native ability, represented by lower SAT-Math scores, had found ways to succeed by the time they reached AAE 35200, a course for third-year engineering students. Although SAT-Math score is an excellent indicator of the academic success for first-year undergraduate students, ${ }^{19,} 20$ it may be less relevant for more advanced students.

The survey also included open-ended qualitative questions about why students chose to attend the supervised homework sessions. Many students indicated that the supervised homework sessions were helpful. Some students also indicated that they learned the most from the homework sessions since they were really trying hard to solve the homework problems, whereas during the lecture, the new materials were simply delivered to the students without practical applications. Other students indicated that the homework was not difficult to complete on their own, so they did not feel the need to attend the supervised homework sessions. Upon reviewing these students' attributes, we found that these students were in the high SAT-Math $(780-800)$ group. This qualitative finding confirms the analysis based on the experimental results shown in Fig. 1.

\section{Conclusions and Future Directions}

From this SoTL project, our intention was to demonstrate that supervised homework sessions had a positive impact on the academic performance of the students. As we analyzed the data, we understood that the homework session had the most positive impact on the homework performance of the students with lower native ability. Also, the study revealed that the exam scores were not correlated with SAT-Math scores as we suspect that the students with lower native ability have found some sort of coping mechanism by the time they reach the 300-level engineering course. We are currently gathering data from the Spring 2016 offering of AAE 35200. As we accumulate more data, we will be able to more accurately determine the effectiveness of the supervised homework sessions for different groups of students, such as women.

\section{References}

${ }^{1}$ M. C. Loui, B. A. Robbins, E. C. Johnson and N. Venkatesan, "Assessment of Peer-led Team Learning in an Engineering Course for Freshmen," International Journal of Engineering Education, vol. 29, no. 6, pp. 14401455, 2013.

${ }^{2}$ L. T. Tien, V. Roth and J. A. Kampmeier, "Implementation of a Peer-Led Team Learning Instructional Approach in an Undergraduate Organic Chemistry Course," Journal of Research in Science Teaching, vol. 39, no. 7, pp. 606-632, 2002. 
${ }^{3}$ W. K. Born, W. Revelle and L. H. Pinto, "Improving Biology Performance with Workshop Groups," Journal of Science Education and Technology, vol. 11, no. 4, pp. 347-365, 2002.

${ }^{4}$ S. C. Hockings, K. J. DeAngelis and R. F. Frey, "Peer-Led Team Learning in General Chemistry: Implementation and Evaluation," Journal of Chemical Education, vol. 85, no. 7, pp. 990-996, 2008.

${ }^{5}$ S. Brown and C. Poor, "In-Class Peer Tutoring: A Model for Engineering Instruction," International Journal of Engineering Education, vol. 26, no. 5, pp. 1111-1119, 2010.

${ }^{6}$ T. J. Webster and K. C. Dee, "Supplemental Instruction Integrated Into an Introductory Engineering Course," Journal of Engineering Education, vol. 87, no. 4, pp. 377-383, 1998.

${ }^{7}$ R. Jacquez, V. G. Gude, A. Hanson, M. Auzenne and S. Williamson, "Enhancing Critical Thinking Skills of Civil Engineering Students through Collaborative Learning Methods in Supplemental Instruction," Proceedings of the 2007 American Society for Annual Conference \& Exposition, pp. 12.664.1 - 12.664.16, 2007.

${ }^{8}$ C. Blat, S. Myers, K. Nunnally and P. Tolley, "Successfully Applying the Supplemental Instruction Model to Sophomore-level Engineering Courses," Proceedings of the 2001 American Society for Engineering Education Annual Conference \& Exposition, pp. 6.911.1 - 6.911.12, 2001.

${ }^{9}$ M. Prince, "Does Active Learning Work? A Review," Journal of Engineering Education, vol. 93, no. 3, pp. 223231, 2004.

${ }^{10}$ D. Montfort, S. Brown and D. Pollock, "An Investigation of Students' Conceptual Understanding in Related Sophomore to Graduate-Level Engineering and Mechanics Courses," Journal of Engineering Education, pp. 111-129, 2009.

${ }^{11}$ S. Brown, D. Montfort and K. Findley, "Student Understanding of States of Stress in Mechanics of Materials," ASEE 2007 Annual Conference \& Exposition, pp. 12.1318.1 - 12.1318.14, 2007.

${ }^{12}$ S. Brown, D. Lewis, D. Montfort and R. L. Borden, "The Importance of Context in Students' Understanding of Normal and Shear Stress in Beams," ASEE 2011 Annual Conference \& Exposition, pp. 22.1471.1 22.1471.12, 2011.

${ }^{13}$ C. T. Sun, Mechanics of Aircraft Structures, 2nd Edition, Hoboken, N.J.: John Wiley \& Sons, 2006.

${ }^{14}$ R. A. Thompson and B. L. Zamboanga, "Academic Aptitude and Prior Knowledge as Predictors of Student Achievement in Introduction to Psychology," Journal of Educational Psychology, vol. 96, no. 4, pp. 778-784, 2004.

${ }^{15}$ K. G. Lewis, "Using Midsemester Student Feedback and Responding to It," New Directions for Teaching and Learning, vol. 2001, no. 87, pp. 33-44, 2001.

${ }^{16}$ N. J. Dorans, "Correspondences between ACT and SAT I Scores," [Online]. Available: https://www.ets.org/Media/Research/pdf/RR-99-02-Dorans.pdf. [Accessed 3 January 2016].

${ }^{17}$ W. J. Camara and G. Echternacht, "The SAT I and High School Grades: Utility in Predicting Success in College (College Board Research Note-10)," College Entrance Examination Board, New York, 2000.

${ }^{18}$ G. Zhang, T. J. Anderson, M. W. Ohland and B. R. Thorndyke, "Identifying Factors Influencing Engineering Student Graduation: A Longitudinal and Cross-Institutional Study," Journal of Engineering Education, vol. 93, no. 4, pp. 313-320, 2004.

${ }^{19}$ K. D. Mattern, B. F. Patterson and J. L. Kobrin, "The Validity of SAT Scores in Predicting First-Year Mathematics and English Grades (College Board Research Report 2012-1)," College Board, New York, 2012.

${ }^{20}$ C. P. Veenstra, E. L. Dey and G. D. Herrin, "Is Modeling of Freshman Engineering Success Different from Modeling of Non-Engineering Success?," Journal of Engineering Education, vol. 97, no. 4, pp. 467-479, 2008. 